Proceedings of Anticancer Research

Research Article

\title{
Clinical Significance of Preoperative Nutritional Risk Screening and Support in the Perioperative Period of Gastric Cancer Patients
}

Defu Tian*, Chao Yin, Bing Ren, Puli Zhu

The Fourth People's Hospital of Shaanxi Province, China

[Abstract] Objective: To investigate the clinical value of using preoperative nutritional risk screening and support in gastric cancer patients. Methods: In this paper, 70 gastric cancer patients selected from July 2017 to July 2020 treated in our hospital were grouped concerning the lottery method, and the reference group $(n=35)$ used conventional nutritional support, while the experimental group $(n=35)$ used preoperative dietary risk screening and support, comparing the clinical treatment differences between gastric cancer patients in the experimental group and the reference group. Results: After the intervention, $\operatorname{IgA}, \operatorname{IgM}, \operatorname{IgG}$, serum albumin, complication rate, NRS score, hospitalization time and anal exhaust time of gastric cancer patients in the experimental group were compared with those in the reference group, $\mathrm{P}<0.05$, and there was statistical validation analysis significance between the data indicators. $\mathrm{P}<0.05$ for the comparison of IgA, IgM, IgG, serum albumin after the intervention and pre-intervention for gastric cancer patients in the experimental group and the reference group, with statistical validation analysis significance between the data indicators. Conclusion: Preoperative nutritional risk screening and support is of significant value in gastric cancer patients and can improve patients' nutritional status.

Keywords: Preoperative nutritional risk screening; Gastric cancer patients; Perioperative period; Clinical significance.

Publication date: September, 2020

Publication online: 30 September, 2020

*Corresponding author:Defu Tian,tiandefu_5@sina.com

Gastric cancer is a common and frequent clinical malignant tumor disease, with the highest incidence and mortality rate among digestive tract tumors, and it has a severe impact on people's health and life safety. According to data, gastric cancer patients often suffer from malnutrition, which increases the mortality rate and complication rate after surgery. The nutritional clean-up of perioperative cancer patients is significantly related to their survival and quality of life ${ }^{[1,2]}$. The better the perioperative dietary status of the patient, the higher the postoperative survival rate, and the lower the mortality and complications. Preoperative nutritional support is, in fact, the key to recovery and its management of complications. This presentation describes the clinical value of using preoperative dietary risk screening and support in 70 patients with gastric cancer between July 2017 and July 2020.

\section{Information and methodology}

\subsection{Basic information}

In this paper, we calculated the objective of the study, that is, 70 gastric cancer patients who participated in the diagnosis and treatment of our hospital from July 2017 to July 2020. In the reference group, there were 35 cases, 20 male patients and 15 female patients, with a median age of (50.54 \pm 2.18$)$ years and an upper limit of 73 years and a lower limit of 33 years, and the depth of invasion was: $7 \mathrm{~T} 1$ patients, $7 \mathrm{~T} 2$ patients, $12 \mathrm{~T} 3$ patients and $9 \mathrm{~T} 4$ patients. In the experimental group, there were 35 cases, 21 male patients and 14 female patients, with a median age of $(51.55 \pm 3.55)$ years and an upper limit of 74 years and a lower limit of 34 years, ans the depth of invasion was: $6 \mathrm{~T} 1$ patients, $8 \mathrm{~T} 2$ patients, 10 $\mathrm{T} 3$ patients and $11 \mathrm{~T} 4$ patients. The comparison and analysis of the general data of gastric cancer patients in the reference group and the experimental group showed 
that $\mathrm{P}>0.05$, and there was no confirmative significance between the statistical indicators.

Inclusion criteria: (1) patients enrolled in the study were diagnosed with gastric cancer by pathological testing and their gastroscopy, meeting the diagnostic tests in the 8th edition of Surgery; (2) preoperative CT and other relevant examinations were free of distant tumor metastasis, and there were no gastric cancer complications such as upper gastrointestinal bleeding and its pyloric obstruction; (3) patients and their families voluntarily participated in the study after understanding the consent form for this surgery, and the referral to the medical ethics committee was accepted.

Exclusion criteria: (1) patients with diabetes mellitus were excluded from the study; (2) patients with intestinal obstruction were excluded from the study; (3) patients with cholecystitis were excluded from the study, and (4) patients with lung function intolerant to surgery were excluded from the study.

\subsection{Methodology}

The reference group carries out routine nutritional support, the experimental group carries out preoperative dietary risk screening and support, selects professionally trained medical and nursing staff to carry out the intervention, follows the NRS 2002 scoring form to carry out NRS assessment for patients admitted to the hospital, if the patient's body mass index, height, etc. cannot be accurately detected, it is necessary to determine their nutrition and condition through the albumin level $(<30 \mathrm{~g} / \mathrm{L})$ index. If the NRS score is more than 3 , the patient is at risk of malnutrition. The relevant personnel are required to implement prophylactic enteral nutrition support for the patient: if the patient's NRS score is less than 3, the patient is not at risk of malnutrition, and the evaluation should be carried out again every other week.

Patients should be given preoperative nutritional support to eat a semi-liquid diet without residue and high in protein. In the experimental group, if the NRS score exceeds $3,1,500 \mathrm{~mL}$ of Risin is administered orally in addition to three meals a day.

\subsection{Observational indicators}

The experimental and reference groups were observed to calculate $\operatorname{IgA}, \operatorname{IgM}, \operatorname{IgG}$, serum albumin, NRS score, length of hospital stay, anal exhaust time, and complication rate.

\subsection{Statistical data methods}

In this paper, the data of 70 gastric cancer patients were analyzed by SPSS21.0 for Windows software package, and the counting data (complication rate) and measurement data (IgA, IgM, IgG, serum albumin, NRS score, hospitalization time, anal exhaust time) were expressed as percentages ( $\mathrm{n}, \%)$ and mean differences, and the test of karyotype and t-test was carried out. The statistical validation analysis of the significance of the data indicators was carried out.

\section{Result}

\subsection{Computational analysis of NRS scores, length of hospital stay, and anal exhaust time in the reference and experimental groups}

NRS score, hospitalization time and anal exhaust time of gastric cancer patients in the experimental group were compared with those in the reference group, $\mathrm{P}<0.05$, and there was statistical validation analysis significance between the data indicators.

Table 1. Comparison of NRS score, hospitalization time and anal exhaust time between the reference group and the experimental group

\begin{tabular}{ccccc}
\hline Grouping & Instances & NRS score & $\begin{array}{c}\text { Length of } \\
\text { hospital } \\
\text { stay (d) }\end{array}$ & $\begin{array}{c}\text { Anal exhaust } \\
\text { time (d) }\end{array}$ \\
\hline $\begin{array}{c}\text { Experimental } \\
\text { group } \\
\begin{array}{c}\text { Reference } \\
\text { group }\end{array}\end{array}$ & 35 & $3.11 \pm 0.11$ & $13.21 \pm 1.22$ & $3.21 \pm 0.31$ \\
X2 & 35 & $3.66 \pm 0.12$ & $17.21 \pm 0.22$ & $5.31 \pm 0.25$ \\
P & 19.9882 & 19.0890 & 31.1962 \\
\hline
\end{tabular}

\subsection{Computational analysis of IgA, IgM, IgG} and serum albumin in the reference and experimental groups

The comparison of $\operatorname{IgA}$, IgM, IgG and serum albumin between patients with gastric cancer in the experimental group and those in the reference group before the intervention, $\mathrm{P}>0.05$, no statistical analysis significance between the data indicators; after the invasion, the comparison of IgA, IgM, IgG and serum albumin between patients with gastric cancer in the experimental group and those in the reference group, $\mathrm{P}<0.05$, statistical verification analysis significance between the data indicators; Patients' IgA, IgM, IgG, and serum albumin in the experimental group and the control group after the intervention compared with those before the invasion, $\mathrm{P}<0.05$, and there was statistical validation analysis significance between the data indicators. 
Table 2. Comparison of $\operatorname{IgA}$, IgM, IgG and serum albumin between the control group and the experimental group

\begin{tabular}{|c|c|c|c|c|c|}
\hline Grouping & Instances & $\operatorname{IgA}(\mathrm{g} / \mathrm{L})$ & $\operatorname{IgM}(g / L)$ & $\operatorname{IgG}(\mathrm{g} / \mathrm{L})$ & $\begin{array}{l}\text { Blood serum albumin } \\
(\mathrm{g} / \mathrm{L})\end{array}$ \\
\hline Experimental group & 35 & & & & \\
\hline Pre-intervention & & $2.11 \pm 0.33$ & $1.11 \pm 1.18$ & $10.22 \pm 3.32$ & $35.55 \pm 2.15$ \\
\hline Post-intervention & & $2.33 \pm 0.12 * \#$ & $1.00 \pm 0.15^{* \#}$ & $8.88 \pm 0.13 * \#$ & $35.22 \pm 0.31 * \#$ \\
\hline Reference grouP & 35 & & & & \\
\hline Pre-intervention & & $2.12 \pm 0.21$ & $1.114 \pm 1.16$ & $10.22 \pm 3.22$ & $35.33 \pm 2.22$ \\
\hline pre-intervention & & $1.82 \pm 0.03 \#$ & $1.21 \pm 0.21 \#$ & $8.01 \pm 0.11 \#$ & $31.21 \pm 0.32 \#$ \\
\hline
\end{tabular}

Note: compare with reference group $* \mathrm{P}<0.05$, compare with pre-intervention $\# \mathrm{P}<0.05$

Table 3. Comparison of complication rate between the reference group and experimental group

\begin{tabular}{|c|c|c|c|c|c|c|}
\hline Grouping & Instances & $\begin{array}{l}\text { Urinary tract } \\
\text { infection }\end{array}$ & Lung infection & $\begin{array}{l}\text { Anastomotic } \\
\text { infection }\end{array}$ & $\begin{array}{l}\text { Gastric drainage } \\
\text { disorder }\end{array}$ & Complication rate \\
\hline $\begin{array}{l}\text { Experimental } \\
\text { group }\end{array}$ & 35 & 0 & 0 & 1 & 1 & $5.71 \%$ \\
\hline Reference group & 35 & 1 & 1 & 4 & 3 & $25.71 \%$ \\
\hline $\mathrm{X} 2$ & & & & & & 5.2851 \\
\hline$P$ & & & & & & 0.0215 \\
\hline
\end{tabular}

\subsection{Computational analysis of complication rates in the reference and experimental groups}

The complication rate of gastric cancer patients in the experimental group was $5.71 \%$ compared with $25.71 \%$ in the reference group, $\mathrm{P}<0.05$, and there was statistical significance between the data and indexes.

\section{Discussion}

The majority of patients admitted with gastric cancer are frequently associated with malnutrition, and a large number of data studies have shown that nutrition is a key influential factor in triggering immune dysfunction in cancer patients ${ }^{[3,4]}$. The physiological and pathological changes caused by malnutrition generally tend to lead to higher mortality and complication rates in patients after surgery. The reasons for the occurrence of malnutrition cover the following aspects: firstly, the patient's dietary intake is inadequate, resulting in decreased appetite. Patients are more prone to illness due to gastric discomfort and their decreased acidity or pain. For patients with pyloric obstruction, vomiting often occurs, which in turn aggravates malnutritionsecondly, hematopoietic insufficiency and chronic blood loss. Patients develop anemia due to impaired absorption and utilization of vitamins and their iron and reduced levels of hemoglobin synthesis ${ }^{[5]}$. Third, elderly patients or those with other chronic diseases are prone to malnutrition. Fourthly, patients with gastric cancer lack nutrition and have different energy consumption from simple starvation. Patients have a lower metabolic rate under starvation condition, and if they cannot consume enough calories, their energy consumption will be reduced. Since gastric cancer patients have an inadequate dietary intake, the metabolic rate increases despite the reduced activity level. Due to the competition for nutrition between the patient and the tumor, changes may occur in gastric mucosa cells in the absence of diet, which may reduce the functionality of the gastrointestinal tract and multiply anaerobic bacteria, resulting in poor absorption of proteins, carbohydrates and fats, efficiently inducing hypoproteinemia and hypovolemia, which not only increases the risk of patients but also decreases their resistance, therefore, nutritional risk assessment is particularly important ${ }^{[6]}$.

The NRS2002 (Nutritional risk screening 2002, NRS2002) is the 2002 European Society for Parenteral and Enteral Nutrition (ESPEN) recommended a screening tool that meets the nutritional risk of adult inpatients, which is assessed in four main areas, including recent changes in body mass, anthropometric measurements, disease severity and their dietary intake [7].

This data study shows that after the intervention of the experimental group of gastric cancer patients IgA, IgM, IgG, serum albumin, complication rate, NRS score, hospitalization time, anal exhaust time compared with the reference group of data of the experimental 
group and the reference group of gastric cancer patients compared with the pre-intervention, $\mathrm{P}<0.05$, there is a statistical validation analysis between data indicators Significance.

$\mathrm{Yu}$ Xiaocui showed that the complication rate of gastrointestinal cancer patients in the observation group was lower than that of the control group, $\mathrm{P}<0.05$, and there was statistical validation analysis significance between the data indicators ${ }^{[8]}$. It was confirmed that preoperative nutritional risk screening was beneficial in reducing complications.

Taken together, the clinical value of using preoperative nutritional risk screening and support was more significant compared with conventional dietary support in patients with gastric cancer.

\section{References}

[1] Ye X. Assessment and analysis of the preoperative nutritional risk of gastric cancer patients by the Nutrition Risk Screening 2002 scoring system[J]. China Rural Health,2019,11(18):50.
[2] He YR. Preoperative nutritional risk screening and analysis of nursing countermeasures in gastrointestinal cancer patients[J]. China Health Care Nutrition,2019,29(16):188.

[3] Guo F, Wu XL, Zhang XY, et al. Study on perioperative nutritional risk screening and dietary support investigation in gastric cancer patients[J]. China Medical Journal, 2020, 17(14):110113.

[4] Dou XY, Zhou XM. Preoperative nutritional risk screening and nursing countermeasures analysis in gastrointestinal cancer patients[J]. Health Care Guide, 2018(35): 129.

[5] Guo F, Wu XL, Zhang XY, et al. Preoperative nutritional risk screening in gastric cancer surgery[J]. Modern Medicine, 2017,45(11):1554-1557.

[6] Liu F, Wu D. The role of preoperative nutritional risk screening in gastric cancer patients and its impact on the incidence of postoperative complications[J]. Journal of Guangxi Medical University,2017,34(11):1594-1597.

[7] Han F, Bian XJ, Chen DY, et al. Comparison of the applicability of different nutritional screening methods to patients with gastrectomy for gastric cancer[J]. Pharmacy and Clinical Research,2019,27(6):449-453.

[8] Yu XC. Preoperative nutritional risk screening and nursing analysis of patients with gastrointestinal cancer[J]. Electronic Journal of Clinical Medical Literature, 2017, 4(83): 1633116333 . 